\title{
Precedent Comparison in the Precedent Model Formalism: A Technical Note
}

\author{
Heng ZHENG ${ }^{\mathrm{a}, 1}$, Davide GROSSI ${ }^{\mathrm{a}, \mathrm{b}}$ and Bart VERHEIJ ${ }^{\mathrm{a}}$ \\ ${ }^{a}$ University of Groningen, The Netherlands \\ ${ }^{\mathrm{b}}$ University of Amsterdam, The Netherlands
}

\begin{abstract}
We outline a formalization of precedent comparison in the precedent model formalism.
\end{abstract}

Keywords. case-based reasoning, precedents, precedent comparison

\section{Introduction}

Case-based reasoning allows for a form of analogical reasoning [1], and a core issue is how to make decisions for a current case by comparing precedents. Building on [2, $3,4]$, we formalize precedent comparison in case-based reasoning within the precedent model formalism [4]. With the definitions and properties shown below, we show that our approach has the potential to offer a novel angle on case-based reasoning.

\section{Precedent comparison in the precedent model formalism}

The formalism introduced in this paper uses a propositional logic language $L$ generated from a set of propositional constants. We fix language $L$. We write $\neg$ for negation, $\wedge$ for conjunction, $\vee$ for disjunction, $\leftrightarrow$ for equivalence. The associated classical, deductive, monotonic consequence relation is denoted $\vDash$.

Precedents consist of factors and outcomes. Both factors and outcomes are literals. A literal is either a propositional constant or its negation. We use $F \subseteq L$ to represent a set of factors, $O \subseteq L$ to represent a set of outcomes. The sets $F$ and $O$ are disjoint and consist only of literals. If a propositional constant $p$ is in $\mathrm{F}$ (or $\mathrm{O}$ ), then $\neg p$ is also in $\mathrm{F}$ (respectively in $\mathrm{O}$ ). A factor represents an element of a case, namely a factual circumstance. Its negation describes the opposite fact. For instance, if a factor $\varphi$ is "A is a bad employee", then its negation $\neg \varphi$ is "A is not a bad employee". In our approach, factors are meant to represent generalized case facts relevant to the outcome of the case decision. However, unlike in CATO [1], our use of factors does not assume that factors favor a side of the decision, either pro-plaintiff or pro-defendant, as such an assumption is not needed for our logical definitions of precedent comparison. Unlike HYPO [1], our factors do not

\footnotetext{
${ }^{1}$ Corresponding Author: Heng Zheng, University of Groningen, Nijenborgh 9, 9747 AG Groningen, The Netherlands; E-mail: h.zheng@rug.nl.
} 
come with a dimension that can express a magnitude. An outcome always favors a side in the precedent, its negation favors the opposite side. For instance, an outcome $\omega$ is "A is dismissed", its negation $\neg \omega$ is "A is not dismissed".

A precedent is a logically consistent conjunction of factors and outcomes. A precedent containing an outcome is a proper precedent. A precedent without an outcome, is a situation representing a current case.

Definition 1 (Precedents) $A$ precedent is a logically consistent conjunction of distinct factors and outcomes $\pi=\varphi_{0} \wedge \varphi_{1} \wedge \ldots \wedge \varphi_{m} \wedge \omega_{0} \wedge \omega_{1} \wedge \ldots \wedge \omega_{n-1}$, where $m$ and $n$ are non-negative integers. We say that $\varphi_{0}, \varphi_{1}, \ldots, \varphi_{m}$ are the factors of $\pi, \omega_{0}, \omega_{1}, \ldots, \omega_{n-1}$ are the outcomes of $\pi$. If $n=0$, then we say that $\pi$ is a situation with no outcomes, otherwise $\pi$ is a proper precedent.

Notice that both $m$ and $n$ can be equal to 0 . When $m=0$, there is one single factor. When $n=0$, the precedent has no outcome and the empty conjunction $\omega_{0} \wedge \ldots \wedge \omega_{n-1}$ is equivalent to $T$. We do not assume that the negation of a factor holds when the factor does not occur in the precedent.

Notions of comparing precedents in case-based reasoning include analogies, distinctions and relevances expressed by general logical formulas, not only factors or outcomes. Analogies between two precedents are the formulas that follow logically from both precedents. Distinctions are the unshared formulas between two precedents, that only follow logically from one of the precedents and its negation is logically implied by the other precedent. Relevances are the unshared formulas between two precedents, that are relevant to the analogies and distinctions between them. These formulas only follow from one of the precedents, but both themselves and their negation are not logically implied by the other one.

Definition 2 (Analogies, distinctions and relevances) Let $\pi, \pi^{\prime} \in L$ be two precedents, we define:

1. a sentence $\alpha \in L$ is an analogy between $\pi$ and $\pi^{\prime}$ if and only if $\pi \vDash \alpha$ and $\pi^{\prime} \vDash \alpha$. A most specific analogy between $\pi$ and $\pi^{\prime}$ is an analogy that logically implies all analogies between $\pi$ and $\pi^{\prime}$.

2. a sentence $\delta \in L$ is a distinction in $\pi$ with respect to $\pi^{\prime}$ ( $\pi-\pi^{\prime}$ distinction) if and only if $\pi \vDash \delta$ and $\pi^{\prime} \vDash \neg \delta$. A most specific $\pi$ - $\pi^{\prime}$ distinction is a distinction that logically implies all $\pi-\pi^{\prime}$ distinctions.

3. a sentence $\rho \in L$ is a relevance in $\pi$ with respect to $\pi^{\prime}$ ( $\pi-\pi^{\prime}$ relevance) if and only if $\pi \vDash \rho, \pi^{\prime} \not \models \rho$ and $\pi^{\prime} \not \forall \neg \rho$. $\rho$ is a proper $\pi$ - $\pi^{\prime}$ relevance if and only if $\rho$ is a $\pi-\pi^{\prime}$ relevance that logically implies the most specific analogy between $\pi$ and $\pi^{\prime}$. A most specific $\pi-\pi^{\prime}$ relevance is a relevance that logically implies all $\pi-\pi^{\prime}$ relevances.

Both $\pi-\pi^{\prime}$ distinctions and $\pi^{\prime}-\pi$ distinctions are called the distinctions between $\pi$ and $\pi^{\prime}$. Both $\pi$ - $\pi^{\prime}$ relevances and $\pi^{\prime}-\pi$ relevances are called the relevances between $\pi$ and $\pi^{\prime}$. When a most specific analogy/distinction/relevance exists it is by definition unique, and we can refer to it as the most specific analogy/distinction/relevance.

Figure 1 illustrates analogies, distinctions and relevances using Venn diagrams representing sets of worlds in which sentences are true. As shown in Figure 1, for any analogy $\alpha$ between precedents $\pi$ and $\pi^{\prime}$, the sets of $\pi$ and $\pi^{\prime}$ worlds are subsets of the set of $\alpha$ worlds; for any $\pi$ - $\pi^{\prime}$ distinction $\delta$, the $\pi$ worlds are a subset of the $\delta$ worlds, while the $\pi^{\prime}$ worlds and the $\delta$ worlds are disjoint; for any $\pi$ - $\pi^{\prime}$ relevance $\rho$, the $\pi$ worlds are 

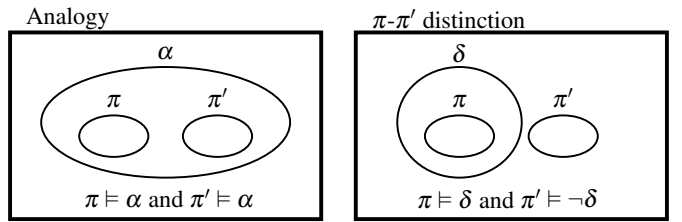

$\pi-\pi^{\prime}$ relevance

Figure 1. Precedent comparison illustrated by worlds in which sentences are true

a subset of the $\rho$ worlds, while the $\pi^{\prime}$ worlds and the $\rho$ worlds are not subsets of each other and the intersection of the $\pi^{\prime}$ worlds and the $\rho$ worlds are always not empty.

The following proposition shows properties of analogies, distinctions and relevances between precedents.

Proposition 1 Let $\pi, \pi^{\prime} \in L$ be precedents. Then the following hold:

1. The most specific analogy between $\pi$ and $\pi^{\prime}$ always exists and is logically equivalent to $\pi \vee \pi^{\prime}$;

2. There exists a distinction between $\pi$ and $\pi^{\prime}$ if and only if $\pi \wedge \pi^{\prime} \vDash \perp$; If a $\pi-\pi^{\prime}$ distinction exists, then the most specific $\pi-\pi^{\prime}$ distinction exists and is logically equivalent to $\pi$;

3. The most specific $\pi-\pi^{\prime}$ relevance does not always exist;

4. If the most specific $\pi-\pi^{\prime}$ distinction exists, then the most specific $\pi$ - $\pi^{\prime}$ distinction logically implies each proper $\pi-\pi^{\prime}$ relevance. Each proper $\pi-\pi^{\prime}$ relevance logically implies the most specific analogy between $\pi$ and $\pi^{\prime}$.

Proof. Let $\pi, \pi^{\prime} \in L$ be precedents. For Property 1, by Definition 2, for any analogy $\alpha, \pi \vDash \alpha$ and $\pi^{\prime} \vDash \alpha$. By propositional logic it follows that any analogy $\alpha$ is logically implied by $\pi \vee \pi^{\prime}$. By Definition 2, $\pi \vee \pi^{\prime}$ is a most specific analogy. For Property 2, assume a $\pi$ - $\pi^{\prime}$ distinction $\delta$ exists. By Definition $2, \pi \vDash \delta$ and $\pi^{\prime} \vDash \neg \delta$. It follows by propositional logic that $\pi \wedge \pi^{\prime} \vDash \perp$. If $\pi \wedge \pi^{\prime} \vDash \perp$, by propositional logic $\pi^{\prime} \vDash \neg \pi$. By Definition 2 and propositional logic, $\pi$ is therefore the most specific $\pi-\pi^{\prime}$ distinction. For Property 3 , assume language $L$ is generated from $\left\{f_{1}, f_{2}\right\}$. If $\pi=f_{1}, \pi^{\prime}=\neg f_{1}$, the most specific $\pi-\pi^{\prime}$ relevance does not exist. $\pi$ - $\pi^{\prime}$ relevances like $f_{1} \vee f_{2}, f_{1} \vee \neg f_{2}$ cannot be logically implied by a unique $\pi-\pi^{\prime}$ relevance. For Property 4 , by Property 2 if the most specific $\pi$ - $\pi^{\prime}$ distinction exists, it is logically equivalent to $\pi$. By Definition $2, \pi$ logically implies all $\pi-\pi^{\prime}$ relevances, including proper ones, and proper $\pi-\pi^{\prime}$ relevances always logically imply the most specific analogy between $\pi$ and $\pi^{\prime}$.

As shown in Proposition 1, the most specific $\pi$ - $\pi^{\prime}$ distinction is logically equivalent to $\pi$ if it exists. Note that a precedent itself can be a distinction since precedents are formulas, hence themselves represent the most specific distinction. Property 4 in Proposition 1 shows why we have singled out proper relevances: in the formally precise sense of the proposition, they are logically 'in between' the most specific distinction (if it exists) and the most specific analogy.

Two precedents can be compared with a third precedent using the analogy relation defined below, which is based on the shared formulas between precedents. When comparing precedents $\pi$ and $\pi^{\prime}$ in terms of precedent $\pi^{\prime \prime}$, if the most specific analogy between $\pi$ and $\pi^{\prime \prime}$ logically implies the most specific analogy between $\pi^{\prime}$ and $\pi^{\prime \prime}$, then we say that $\pi$ is at least as analogous as $\pi^{\prime}$ with respect to $\pi^{\prime \prime}$. 
Definition 3 (Analogy relation between precedents) Let $\pi, \pi^{\prime}$ and $\pi^{\prime \prime} \in L$ be precedents. We define:

$$
\pi \succeq_{\pi^{\prime \prime}} \pi^{\prime} \text { if and only if } \pi \vee \pi^{\prime \prime} \vDash \pi^{\prime} \vee \pi^{\prime \prime} .
$$

Then we say $\pi$ is at least as analogous as $\pi^{\prime}$ with respect to $\pi^{\prime \prime}$.

As customary, the asymmetric part of the relation is denoted as $\pi \succ_{\pi^{\prime \prime}} \pi^{\prime}$, which means $\pi$ is more analogous than $\pi^{\prime}$ with respect to $\pi^{\prime \prime}$. The symmetric part of the relation is denoted as $\pi \sim \pi^{\prime \prime} \pi^{\prime}$, which means $\pi$ is as analogous as $\pi^{\prime}$ with respect to $\pi^{\prime \prime}$. If it is not the case that $\pi \succeq_{\pi^{\prime \prime}} \pi^{\prime}$ and $\pi^{\prime} \succeq_{\pi^{\prime \prime}} \pi$, then we say $\pi$ and $\pi^{\prime}$ are analogously incomparable with respect to $\pi^{\prime \prime}$.

Proposition 2 Let $\pi, \pi^{\prime}$ and $\pi^{\prime \prime} \in L$ be precedents. Then the following holds:

1. The analogy relation is reflexive and transitive, hence a preorder;

2. $\pi \succeq_{\pi^{\prime \prime}} \pi^{\prime}$ if and only if $\pi \vDash \pi^{\prime} \vee \pi^{\prime \prime}$;

3. If $\pi \succeq_{\pi^{\prime \prime}} \pi^{\prime}$, then $\pi \succeq_{\pi^{\prime}} \pi^{\prime \prime}$ and vice versa;

4. For any $\alpha \in L$, if $\pi \succeq_{\pi^{\prime \prime}} \pi^{\prime}$, and $\alpha$ is an analogy between $\pi^{\prime}$ and $\pi^{\prime \prime}$, then $\alpha$ is also an analogy between $\pi$ and $\pi^{\prime \prime}$.

Proof. For property 1, the analogy relation is reflexive, since $\pi \vee \pi^{\prime \prime} \vDash \pi \vee \pi^{\prime \prime}$. The relation is also transitive because of the transitivity of entailment in propositional logic. Assume $\pi=f_{1} \wedge f_{2}, \pi^{\prime}=f_{1} \wedge f_{3}$ and $\pi^{\prime \prime}=f_{1} \wedge f_{2} \wedge f_{3}, \pi$ and $\pi^{\prime}$ are analogously incomparable with respect to $\pi^{\prime \prime}$, hence the relation is not in general total. For Property 2 , from left to right, by Definition 3 we obtain $\pi \vee \pi^{\prime \prime} \vDash \pi^{\prime} \vee \pi^{\prime \prime}$, and by propositional $\operatorname{logic} \pi \vDash \pi^{\prime} \vee \pi^{\prime \prime}$. From right to left, from $\pi \vDash \pi^{\prime} \vee \pi^{\prime \prime}$ and propositional logic, we obtain $\pi \vee \pi^{\prime \prime} \vDash \pi \vee \pi^{\prime \prime}$, and by Definition $3 \pi \succeq_{\pi^{\prime \prime}} \pi^{\prime}$. Property 3 then follows directly from Property 2. Property 4 follows directly from Definition 2 and 3.

Notice that if $\pi \succeq \pi^{\prime \prime} \pi^{\prime}$, then it is still possible that $\pi \not \forall \pi^{\prime}$ and $\pi \not \forall \pi^{\prime \prime}$. For instance, if $\pi=f_{1}, \pi^{\prime}=f_{1} \wedge f_{2}, \pi^{\prime \prime}=f_{1} \wedge \neg f_{2}$, then we have $\pi \succeq_{\pi^{\prime \prime}} \pi^{\prime}$, but both $\pi^{\prime}$ and $\pi^{\prime \prime}$ are not logically implied by $\pi$. Also notice that if $\pi \succeq \pi^{\prime \prime} \pi^{\prime}$, it cannot be concluded that $\pi \vDash \pi^{\prime}$. For instance, $\pi=f_{1} \wedge f_{2}, \pi^{\prime}=f_{3}$ and $\pi^{\prime \prime}=f_{1}$. In this example, $\pi \succeq_{\pi^{\prime \prime}} \pi^{\prime}$ but $f_{1} \wedge f_{2} \not f_{3}$.

\section{Conclusion}

In this technical note, we showed how to incorporate a form of precedent comparison in the precedent model formalism of [4]. In future work we aim to develop this approach further and use it to represent and reason about actual legal cases.

\section{References}

[1] K. D. Ashley. Artificial Intelligence and Legal Analytics: New Tools for Law Practice in the Digital Age. Cambridge University Press, Cambridge, 2017.

[2] B. Verheij. Formalizing Arguments, Rules and Cases. In Proceedings of the Sixteenth International Conference on Articial Intelligence and Law, ICAIL 2017, pages 199-208. ACM, New York, 2017.

[3] H. Zheng, M. Xiong, and B. Verheij. Checking the Validity of Rule-Based Arguments Grounded in Cases: A Computational Approach. In M. Palmirani, editor, Legal Knowledge and Information Systems. JURIX 2018: The Thirty-first Annual Conference, volume 313, pages 220 - 224. IOS Press, Amsterdam, 2018.

[4] H. Zheng, D. Grossi, and B. Verheij. Case-Based Reasoning with Precedent Models: Preliminary Report. In H. Prakken, S. Bistarelli, F. Santini, and C. Taticchi, editors, Computational Models of Argument. Proceedings of COMMA 2020, volume 326, pages 443-450. IOS Press, Amsterdam, 2020. 